\title{
Segmentation of CT Image using MAP-Model and Simulation Annealing
}

\author{
Md. Gauhar \\ Arefin \\ Dept. of Computer \\ Science \& \\ Engineering, \\ Mawlana Bhashani \\ Science and \\ Technology \\ University, Tangail- \\ 1902, Bangladesh
}

\author{
Mithun Kumar \\ PK. \\ Dept. of Computer \\ Science \& \\ Engineering, \\ Mawlana Bhashani \\ Science and \\ Technology \\ University, Tangail- \\ 1902, Bangladesh
}

\author{
Abu Sayem \\ Mohammad \\ Delowar Hossain \\ Dept. of Computer \\ Science \& \\ Engineering, \\ Mawlana Bhashani \\ Science and \\ Technology \\ University, Tangail- \\ 1902, Bangladesh
}

\author{
Mohammad \\ Motiur Rahman \\ Dept. of Computer \\ Science \& \\ Engineering, \\ Mawlana Bhashani \\ Science and \\ Technology \\ University, Tangail- \\ 1902, Bangladesh
}

\begin{abstract}
Segmentation on Computed Tomography (CT) image of heart and brain can be optimally posed as Bayesian labeling in which the segment of a image is defined as the maximum a posteriori (MAP) probability estimate of the true labeling. The Simulated Annealing (SA) algorithm is used to minimize the energy function associated with MRF posterior distribution function. The goal of this thesis paper is to minimize the energy function using Gaussian distribution and get accurate segmentation by slowly minimize the energy and simultaneously reduce the pixels which have no impact on the image at rapid rate to get the segmentation quickly without degrade the image. The propose algorithm able to get more precise segmentation.
\end{abstract}

\section{Keywords}

Segmentation, Computer Tomography (CT), Maximum a Posteriori probability (MAP), Markov Random Field (MRF), Simulated Annealing (SA) algorithm

\section{INTRODUCTION}

Nowadays medical image segmentation is an important issue in several application of medical image processing and computer vision. It is the process of partitioning an medical image into homogeneous and disjoin region [1][2]. A group of connected pixels in an image which share a common feature is called a homogeneous region. Nowadays, CT (Computed Tomography) Imaging is a prevalent way of realizing human heart and brain and mostly utilized in diagnostics. Due to the nature of the CT image, it is not simple to segment. Segmenting on brain and heart CT image is difficult and sensitive. In designing segmentation algorithms incorporating expert knowledge is conspicuously useful. An improved simulated annealing (SA) segmentation algorithm base on Maximum a posteriori probability (MAP) and Markov random field (MRF) is represented in this paper [3]-[5].

There is a lot of thesis in segmenting heart and brain image have focused on the distribution of pixel intensities in tissue and try to calculate the parameters of their probability distribution functions [6]. One of the general methods in this area is the Byees rule which allow maximizes a posteriori probability of intensities by segmenting or classifying them into various region or tissues [7]. Pixel classes are represented by multivariate Gaussian distributions and a second order MRF model is used. SA algorithm is used due to obtain the MAP estimate.

Our approach suggested that the high resolution, lower noise CT structural information improved segmentation performance. In this paper we improve the work of [8][9] on 2D CT heart and brain image using a MAP-MRF model. Our aim is to get better segmentation with slowly decreasing temperature at simulated annealing algorithm and quickly remove the pixel which are not belong to heart chamber and the region which has much blood.

\section{METHODOLOGY}

\subsection{Preprocessing}

Before segmentation 2D CT image some preprocessing should be done due to better segmentation and avoiding unexpected errors. Skull and non-brain or non-blood tissues and background are removed by some low level threshold and dilation use to ensure all brain tissues or blood of heart are covered by it. To close the holes inside the brain or blood regions some morphological operation should used. The segmentation algorithm are processed when the pixel remain to the brain or blood regions.

\subsection{MAP based Markovian Bayes method}

MRF provides contextual or spatial information into the segmentation algorithm. This models theory is a tool to encode contextual constraints into the prior probability. A complete segmentation based on MRF model is formulated within the Bayesian framework. One of the Markovian property says that, the probability of belonging each pixel to a class with assumption that other pixels appropriate class are known, is equal to the probability of belonging that pixel to the same class with assumption that just its neighbor pixels appropriate class are known [10], can be derived as

$p\left(x_{i} \mid X_{r}, r \neq i\right)=p\left(x_{i} \mid X_{i}, r \in N_{i}\right)$

where $N_{i}$ are the neighbors of pixel $i$. Hence, MRF can be defined with a conditional probability with respect to a neighboring system [11]. 
Bayesian statistics is a fundamental importance of estimation and decision making. Let, consider a random field $\langle X, Y\rangle$ defined on a rectangle lattice $S=\{(i, j): 1 \leq i \leq W, 1 \leq j \leq H\}$. An observed CT image $x=\{x: s \in S\}$ and a configuration $y=\{y: s \in S\}$ are instances of random field $X$ and $Y$, respectively. According to the posteriori probability theory [5][12], the segmentation of optimal configuration $\mathrm{y}^{*}$, which maximizes the posterior probability. We can formalized from Bayes rule

$y^{*}=\arg \max _{y \in N_{z}} \frac{p(x \mid y) p(y)}{p(x)}$

Where $N_{z}$ is the set of all configuration, $p(y)$ is the probability observed image, which dose not vary with respect to any configurations, $p(x, y)$ is the likelihood probability of the observed image on condition of the configuration $z$.

The likelihood probability can be calculate by Gaussian distribution and can be derive as

$$
p(x \mid y)=\arg \max _{s} \frac{1}{\sqrt{(2 \Pi)^{2} \sigma^{2}}} e^{\left(-\frac{(x-\bar{x})^{2}}{2 \sigma^{2}}\right)}
$$

Where $\sigma$ and $x$ are the distribution parameters and the distribution function can be derived by Gibbs function according to Hammersley-Clifford theorem [13]

$p(y)=\frac{1}{Z} e^{\left[-\frac{1}{T} \sum_{s} \sum_{t \in \eta} V\left(y_{s}, y_{t}\right)\right]}$

Where $Z$ is a normalizing constant, $T$ is temperature which controls the sharpness of the distribution. Initial value of $T$ is $1, V\left(y_{s}, y_{t}\right)$ is the potential of the clique $\left\{y_{s}, y_{t}\right\}$ and $\eta$ is the neighborhood of s. $V\left(y_{s}, y_{t}\right)$ can be defined as

$V\left(y_{s}, y_{t}\right)=\left\{\begin{array}{c}1 ; y_{s} \neq y_{t} \\ -1 ; y_{s}=y_{t}\end{array}\right.$

Substituting Eq. (3) and (4) in (2) we have

$$
\left.y^{*}=\arg \max _{y} \frac{1}{Z 2 \Pi \sigma} e^{\left[-\frac{(x-x)^{2}}{2 \sigma^{2}}+U(y)\right.}\right]
$$

The maximization of the above function is equal to the minimization of the following [14].

$$
y^{*}=\arg \max _{y}\left[\alpha\left(\frac{(x-\bar{x})^{2}}{2 \sigma^{2}}\right)+U(y)\right]
$$

where $\frac{(x-\bar{x})^{2}}{2 \sigma^{2}}$ is the Gaussian energy, $U(y)$ is the Gibbs energy and $\alpha$ is a weighting factor.

\subsection{Segmentation}

Segmentation of 2D CT image is done by Simulated Annealing algorithm, which is proposed by Kirkpatrick [4][15]. The SA process consists of melting the system being optimized at a high effective temperature, then lowering the temperature by slow stages until the system freezes and no further changes occur. The Gaussian energy of equation (6) is calculated using expectation-maximization (EM) algorithm with simulated annealing. The steps of SA algorithm are described below:

1. Initialize the temperature $T$.

2. Compute the energy $U$ of the configuration.

3. Use the maximum likelihood estimation [4] to calculate Gaussian distribution parameters.

4. Use the Metropolis sampler [5] with the simulated annealing scheme [3] to refine the configuration and compute the new energy of the perturbed system and evaluate the change in the energy.

$\Delta U=U-U^{\prime}$

5. If $(\Delta U>0)$, accept the perturbed system as the new configuration, Else accept the perturbed system as the new configuration with a probability $\exp (\Delta U$ $/ T(n))$, where

$T(n)=T(n-1) x(\log (C)) / \log (C+n)$

is the annealing temperature sequence, $T(n-1)=1$ is the initial temperature and $\mathrm{n}$ is the iteration number. Here the value of $T(n)$ is dependent on the previous value of $T$ and the logarithm allow slowly deceasing of temperature.

6. Repeat above two steps until get the desire segmentation.

The weighting factor presents the trade-off between the fidelity of the observed image and the smoothness prior of the configuration. It is expected to be high at initial stages of the optimization due to the blood region of CT image can play a dominant role, and slowly smaller in the later state. For better segmentation, consume time or less iteration and by observing the statistical data of images we propose to use equation (10) instate of $\alpha$ in [8].

$\alpha(n)=\alpha(n-1) \times 0.9^{n}$

Here, $\alpha(n-1)$ is the previous value of $\alpha(n)$, which allow deceasing value of present alpha rapidly to get to the desire segmentation.

We use equation (9) to get slow temperature deceasing which helps to avoid error segmentation. On the other hand, we use rapid deceasing of weighting factor to remove non-usable region or pixels. By following these processes we can have better segmentation quickly without have any casualty.

\section{RESULTS}

In 2D CT image segmentation the blood of heart chamber and brain region are main concentration of this thesis. Without losing the important information belong to the CT image and segment the major regions is only focus of the propose algorithm. After remove the background we implement the algorithm of [8] and the propose algorithm on 16 slice of heart and brain CT images, we find out that the resulting image of our proposed algorithm gets better segmentation. In Figure 1 (b) we remove background from the heart CT image and Fig.1(c) - (d) show segmented image at iteration 15 of [8] and segmentation at iteration 6 of proposed algorithm. We can see that the proposed algorithm have better segmentation than implemented one. Our aim is to get better segmentation with slowly decreasing temperature $T$ and quickly remove the pixel which are not belong to heart chamber and the region which has much blood with rapidly decease of weighting factor 


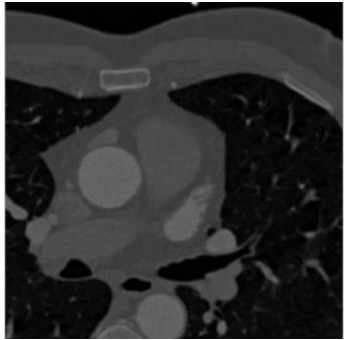

(a)

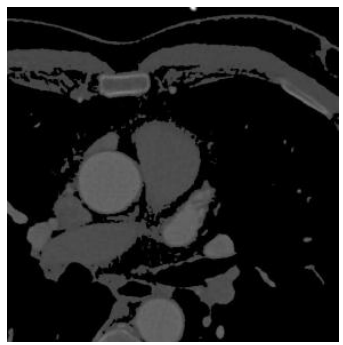

(b)

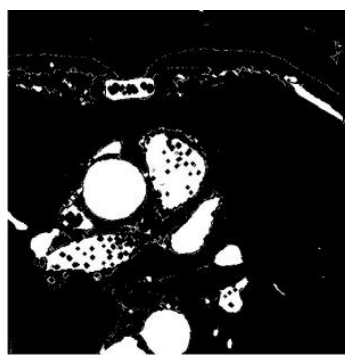

(e)

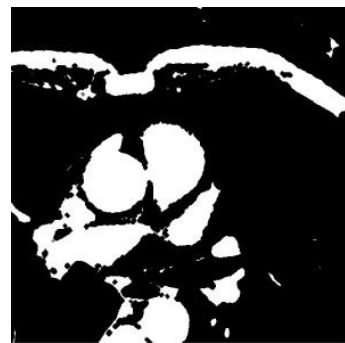

(c)

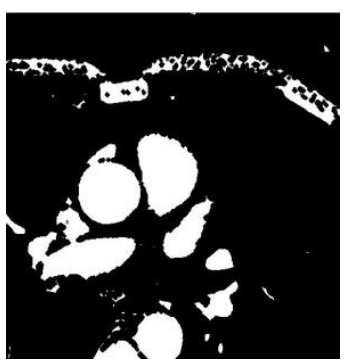

(f)

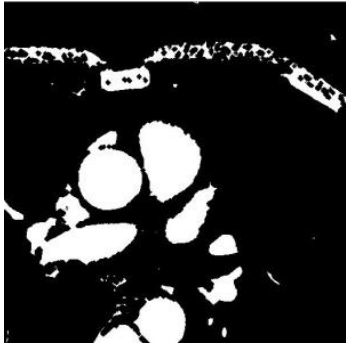

(d)

Figure 1: A slice of heart CT image [512 x 512] and 16-bit of (a) original image, (b) image after removing the background, (c) implemented algorithm's image at iter=15, (d) proposed image at iter=6, (e) implemented algorithm's image at iter=16, (f) proposed image at iter $=7$

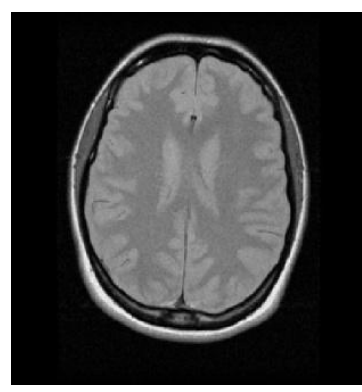

(a)

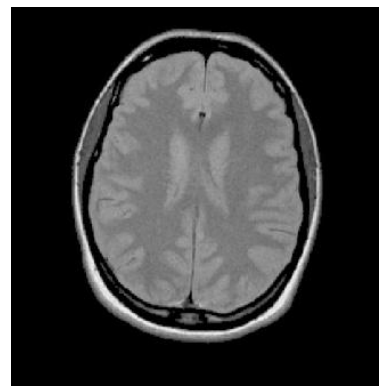

(b)

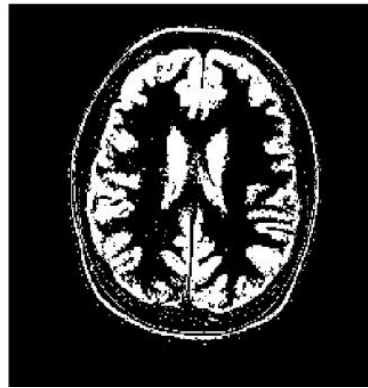

(c)

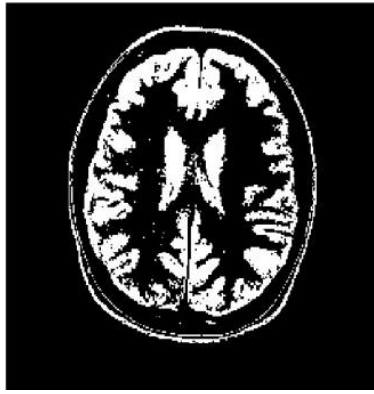

(d)

Figure 2: A slice of brain CT image [256 x 256], 16-bit of (a) original image, (b) image after removing the background, (c) proposed image at iter $=8$, (d) proposed image at iter $=9$

alpha $\alpha(n)$ of equation (10). Fig.1(e)-(f) show the segmentation of implemented and proposed algorithm respectably. The implemented algorithm's image is degraded and not even get the proper segmentation, on the other hand proposed algorithm do get desired result without degrade the image. Fig. 1 is experimented using $\sigma=3.3$, initial $T=1$ and initial $\alpha=0.1$.Figure 2(c) and (d) represent the segmented image of brain at iteration 8 and 9 respectably using $\sigma=3.5$.

Finally, the comparative experiments are carried out on several clinical studies. Observing Fig.1 and 2, it illustrates again that our approach provides more accurate brain and heart CT image segmentation. The statistical observation of $\alpha$ makes the proposed image more efficient then other segmentation process.

\section{CONCLUSION}

We propose a simultaneous process in SA to segment heart and brain CT images based on the MAP-MRF model. By simultaneously decrease temperature and weighting factor we get accurate delineation of segmentation. The propose algorithm require precise value of iteration and $\sigma$. It needs user interaction to get almost perfect segment, but it need short time or small iteration. In some CT image propose algorithm degrade after some iteration.

\section{REFERENCES}

[1] L. Lucchese, S.K. Mitra, "Color Image Segmentation: A State of the Art Survey," (invited paper), Image Processing, Vision and Pattern Recognition, Proc. of Indian National Science Academy, vol. 67, A, No.2, pp. 207-221, March-2001.

[2] H.D. Cheng, X.H. Jiang, Y. Sun and Jingli Wang, "Color Image Segmentation: Advances and Prospects," Pattern Recognition, vol. 34, pp. 2259-2281, 2001.

[3] S. Kirkpatrick, C. D. Gelatt Jr, and M. P. Vecchi, "Optimization by simulated annealing," Science, vol. 220, no. 4598, pp. 671-680, 1983.

[4] H. Deng, and D.A. Clausi, "Unsupervised image segmentation using a simple MRF model with a new implementation scheme," Pattern Recognit., vol. 37, no. 12, pp. 2323-2335, 2004.

[5] S. Geman, D. Geman, "Stochastic relaxation, Gibbs distributions, and the Bayesian restoration of images", 
IEEE Transaction on Pattern Analysis and Machine Intelligence, vol. 6, pp. 721-741, 1984.

[6] Shen S, Szameitat AJ, Sterr A. "An improved lesion detection approach based on similarity measurement between fuzzy intensity segmentation and spatial probability maps. Magnetic Resonance Imaging ". 2010; 28(2):245-54.

[7] Yong Y, Pan L, Chongxun Z, editors “An efficient statistical method for segmentation of single channel brain MRI Computer and Information Technology", 2004. CIT '04. The Fourth International Conference on; 2004 14-16 Sept. 2004.

[8] Xia Y, Wen L, Eberl S, Fulham M, Feng D. "Segmentation of dual modality brain PET/CT images using the MAP-MRF model". Multimedia Signal Processing, 2008 IEEE $10^{\text {th }}$ Workshop on, IEEE. 2008:107-110.

[9] Y. Xia, L. Wen, S. Eberl, M. Fulham, and D. Feng, "Segmentation of brain structures using PET-CT images," in Proc. of the 5th International Conference on Information Technology and Applications in Biomedicine (ITAB 2008), Shenzhen, China, May 30-31, 2008.
[10] Geman S, Geman D, Abend K, Harley T, Kanal L. "Stochastic relaxation, Gibbs distributions and the Bayesian restoration of images". Journal of Applied Statistics.1993; 20(5):25-62.

[11] Stolkin R, Greig A, Hodgetts M, Gilby J. “An EM/EMRF algorithm for adaptive model based tracking in extremely poor visibility". Image and Vision Computing. 2008;26(4):480-95.

[12] S.Z. Li, "Modeling image analysis problems using Markov random fields," in C. R. Rao and D. N. Shanbhag (ed), Stochastic Processes: Modeling and simulation, vol. 20 of Handbook of Statistics. Elsevier Science, 2000, pp. 1-43.

[13] J.M. Hammersley, and P. Clifford, "Markov field on finite graphs and lattices", unpublished, 1971.

[14] Sucheta Panda and P. K. Nanda, "Unsupervised Color Image Segmentation Using Compound Markov Random Field Model" Proc. of International Conference on Pattern Recognition and Machine Intelligence (PReMI09), 16-20 Dec, 2009, IIT Delhi, India.

[15] Y. Xia, D. Feng, and R. Zhao, "Adaptive Segmentation of Textured Images by Using the Coupled Markov Random Field Model,” IEEE Trans. Image Processing, vol. 15, pp.3559-3566, Nov. 2006. 